\title{
Design and implementation of anulus-shaped ciliary structure for four-unit MEMS vector hydrophone
}

\author{
Xiaoyong Zhang ${ }^{1,2, a}$, Nixin Shen ${ }^{1,3, a}$, Qingda $\mathrm{Xu}^{4}$, Yu Pei ${ }^{1}$, Yuqi Lian ${ }^{1}$, Weidong Wang ${ }^{1}$, \\ Guojun Zhang $^{1, *}$, and Wendong Zhang ${ }^{1}$ \\ ${ }^{1}$ State Key Laboratory of Dynamic Testing Technology, Taiyuan 030051, PR China \\ ${ }^{2}$ Department of Intelligence and Automation, Taiyuan University, Taiyuan 030032, PR China \\ ${ }^{3}$ Nanjing North Information Industrialization Group Co., Ltd., Nanjing 211153, PR China \\ ${ }^{4}$ Shanghai Jiao Tong University, Shanghai, 200000, China
}

Received: 22 December 2020 / Accepted: 10 February 2021

\begin{abstract}
Inspired from fish bionics, an anulus-shaped ciliary structure for four-unit MEMS vector hydrophone (AFUVH) is proposed in this paper. It replaces the previous bionic cilium-shaped structure and becomes a novel sensing structure in the four-unit MEMS vector hydrophone (FUVH), which realizes the complete simulation of the fish lateral line neuromasts structurally and functionally. It not only improves the sensitivity of FUVH, but also retains a sufficient bandwidth, which improves the overall performance of FUVH for monitoring remote ships noise. Meanwhile, this paper theoretically analyzes the performance of AFUVH for detecting acoustic signals. ANSYS 17.0 simulation is used to derive the parameters of effective structure and optimum dimension structure of annulus-shaped ciliary structure. Finally, the experimental results show that comparing with cilium-structured FUVH, the sensitivity of AFUVH with effective structure is increased by $3.92 \mathrm{~dB}$, and the sensitivity of AFUVH with optimum dimension structure is increased by $5.87 \mathrm{~dB}$, reaching up to $-177.53 \mathrm{~dB}$.
\end{abstract}

Keywords: Vector hydrophone / sensitivity / bionics / neuromasts

\section{Introduction}

With the development of science and technics, biosystem is one of the main references to create new technologies. Under this background, bionics has been promoted in developing novel sensor designs, investigating new sensor materials and studying sensing principles. Based on the bionics principle, Xue et al. firstly presented the ciliumshaped MEMS vector hydrophone $(\mathrm{CVH})$ to mimick the lateral organs of fish, and its sensitivity reached up to $-197.7 \mathrm{~dB}$ at $1 \mathrm{kHz}(0 \mathrm{~dB}$ re $1 \mathrm{~V} / \mu \mathrm{Pa})$ [1]. Bionic cilia cylinder is used to pick up sound signals, which is a significant sensing part for CVH. Inspired by this bionic principle, Hanasoge et al. proposed a magnetic artificial cilia fabricating method, which is applied to pump and mix microfluidic, and other fluid handing processes [2]. Kottapalli et al. reported a SU-8 3D hair cell structure (Fig. 1a) [3]; Krijnen et al. reported the array of spiral-suspended sensory hairs (Fig. 1b) [4]; Zhang et al. reported the design and analysis of a multiple sensor units vector hydrophone (Fig. 1c) [5].

*Corresponding author: zhangguojun1977@nuc.edu.cn

${ }^{a}$ Xiaoyong Zhang and Nixin Shen contributed equally to this work.
Based on all these above, biomimetic sensors are gradually developing towards miniaturization and arrays. Therefore, North University of China designed and manufactured FUVH based on the research of MUVH. The FUVH design process takes fish lateral line neuromasts as the reference. By simulating this biologic structure and its function, the design purpose of FUVH is to improve the performance of MEMS vector hydrophone for detecting remote ships noise [6]. However, FUVH is still used as a simple combination of four independent cilium MEMS vector hydrophones (CVHs) in current application. Neither the design of sensing structure nor the application methods are innovative. Thus, the advantage of multiple sensing units cannot be fully utilized. Meanwhile, still using previous ciliumshaped sensing structure is unable to achieve the current application purpose of FUVH. From previous research, the other main restriction is that the improvement of sensitivity is counteracted by the expanding bandwidth [7]. Therefore, methods to improve the sensitivity of sensing structures and to ensure the appropriate bandwidth have been presented as follows: Bora et al. reported the HA-MA hydrogel encapsulated PZT sensor that was covered by the dome shaped cupula (Fig. 2a) [8]; Liu et al. presented a "lollipop-shaped" MEMS vector hydrophone, whose cilium structure is integrated with a low density sphere (Fig. 2b2) [9]; Xu et al. 


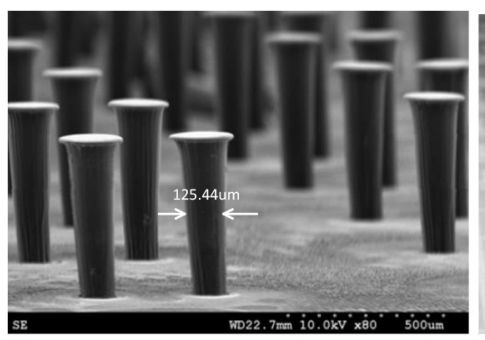

(a)

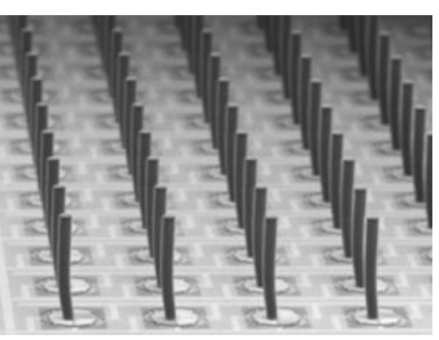

(b)

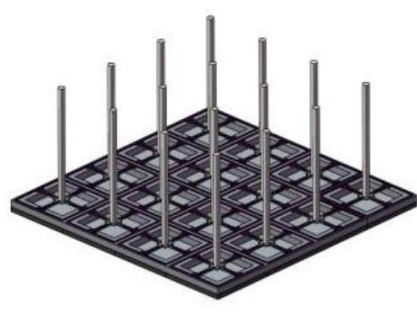

(c)

Fig. 1. Biomimetic sensors based on a bionic cilium.

HA-MA hydrogel dome shaped cupula

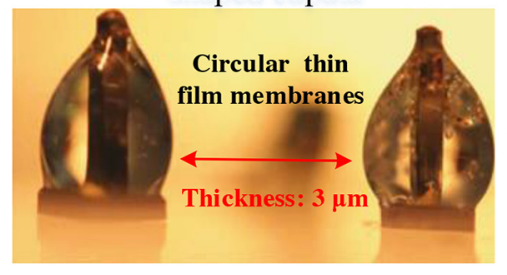

(a)

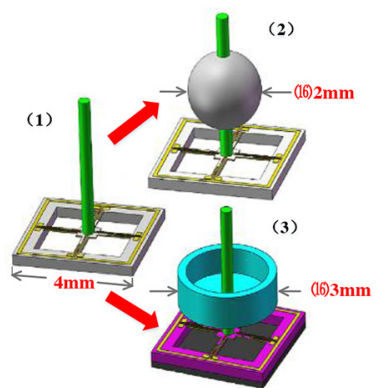

(b)

Fig. 2. Biomimetic sensors based on the expanding sensing areas.

Table 1. Features of different hydrophones.

\begin{tabular}{lllll}
\hline Features & \multicolumn{3}{c}{ Previous works } & This work \\
\cline { 2 - 4 } Hydrophone & Lollipop-shaped & Cup-shaped & FUVH & AFUVH \\
\hline Improvement of sensitivity (compared with CVH) & $10 \mathrm{~dB}$ & $4.3 \mathrm{~dB}$ & $11.8 \mathrm{~dB}$ & $17.67 \mathrm{~dB}$ \\
Natural frequency of FSI & $862 \mathrm{~Hz}$ & $1009.1 \mathrm{~Hz}$ & $1356.07 \mathrm{~Hz}$ & $1216.62 \mathrm{~Hz}$ \\
\hline
\end{tabular}

presented a "cup-shaped" MEMS vector hydrophone, whose main sensing structure is replaced by an artificial "cup" (Fig. 2b3) [7].

Both the design of lollipop-shape and cup-shape are used to mimick the cupula of the lateral line neuromast, which has been proven to enhance the sensitivity by expanding sensing areas. However, for the size restriction of FUVH sensor chip, both the design methods above are no longer fit for FUVH. Based on this principle, the anulusshaped ciliary structure for FUVH is proposed in this paper. This novel structure integrates a low-density rigid ring with previous cilium cylinders, which not only makes four single sensor units of FUVH become an entirety, but also ensures the advantages of sensitivity and working bandwidth. Features of different hydrophones are shown in Table 1.

\section{Bio-inspiration}

From the study of lateral line system, the signal receptors are neuromasts that are mainly used to perceive the flow of water and the movement of the prey target. Taking superficial neuromasts (SNs) as example, SNs are covered by a slender cupula and have bundles of cilia inside. When lateral line system senses signals, the cupula tilts, then the ciliary bundles transmit stimulus signals to sensory cells, and then to nerve fibers [10], as shown in Figure 3a. The innervation structure of SNs is that a nerve innervates a group of adjacent SNs after branching. As shown in Figure 3b, this structure and SN groups constitute the fish lateral line system together. The SEM image of the blind cave fish lateral line neuromast is shown in Figure 3c.

Based on the SNs bionics principle and hydroacoustic propagation theory, the sensing structure of AFUVH is composed of three parts: a solid ring, four cilia cylinders and cross-beams of four sensor units. All three parts are rigidly intergraded to ensure that the force moment is transmitted without any rotation angle. The rigid ring is a simple but effective simulation of the cupula macroscopically. The whole structure accomplishes the simulation of the sensing process, from the movement of cupula to the signal reception of sensory cells. When annulus-shaped ciliary structure receives underwater acoustic signals, the rigid ring firstly drives four cilia cylinders to move. Their movements transfer the force moment to cross-beams, and then induce the deformation of four cross-beams in each unit. Resistances (rate) of the piezoresistors on the cross-beams change with the deformation. Finally, through Wheatstone Bridge, the acoustic signals are converted to the voltage outputs, 


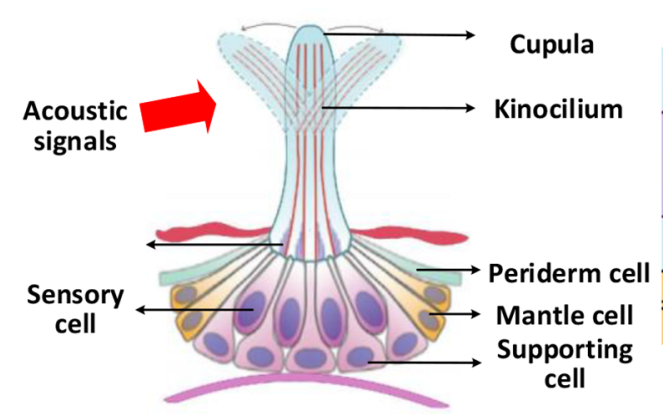

(a)

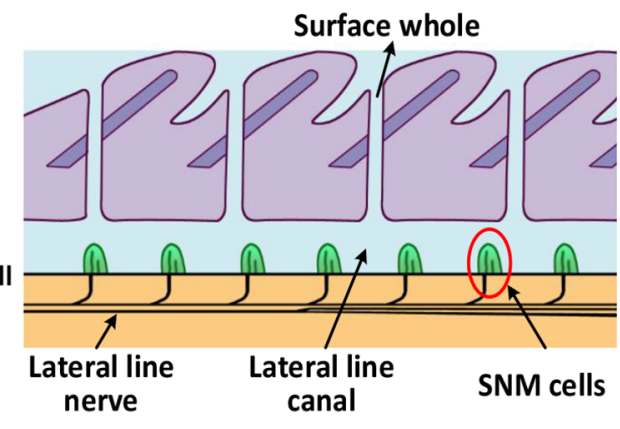

(b)

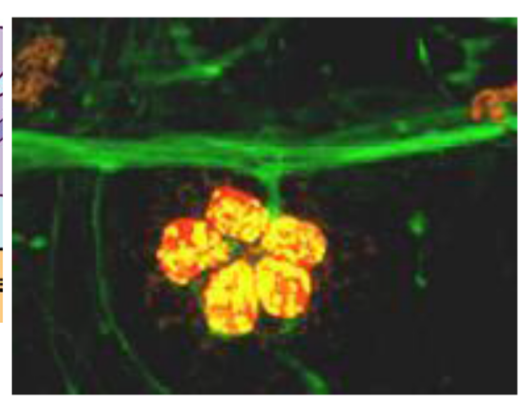

(c)

Fig. 3. (a) Four hair cells occur at the center of a schematic depiction of a single SN, modified after Ref. [11]; (b) Schematic diagram of fish lateral line system; (c) The SEM image of the blind cave fish lateral line neuromast: a neuromast consists of five adjacent sensory cells (yellow) and a lateral nerve (green).

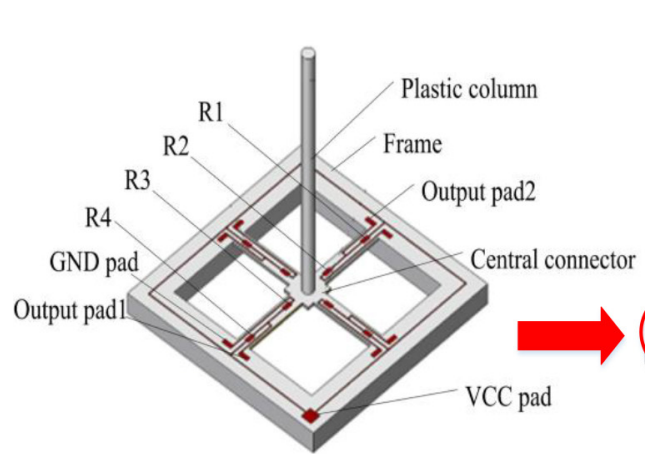

(a)

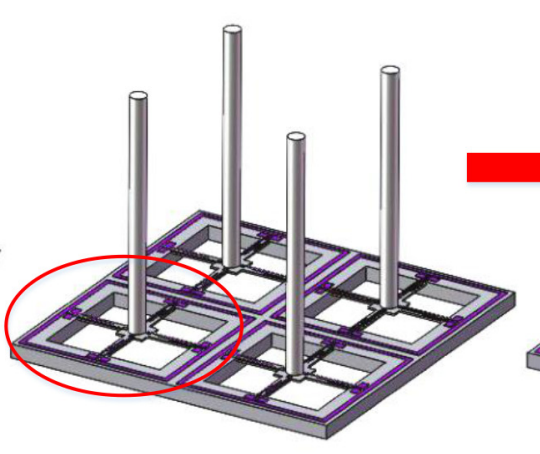

(b)

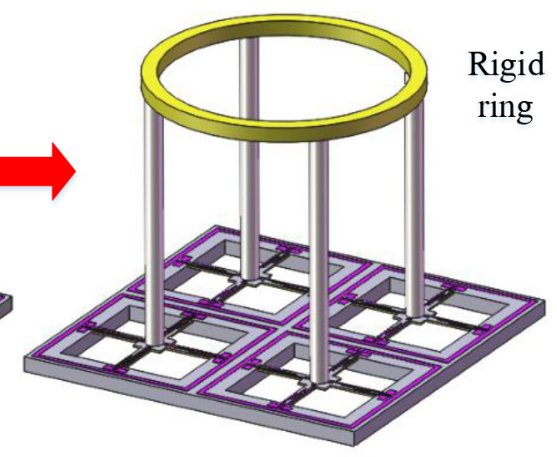

(c)

Fig. 4. (a) Cilium MEMS vector hydrophone (CVH); (b) Four-unit MEMS vector hydrophone (FUVH); (c) Anulus-shaped ciliary structure MEMS vector hydrophone (AFUVH).

Table 2. Material properties.

\begin{tabular}{lllll}
\hline No & Material & Density $\left(\mathrm{kg} \mathrm{m}^{-3}\right)$ & Poisson coefficient & Elastic modulus $(\mathrm{Pa})$ \\
\hline 1 & PE & $0.95 \times 10^{3}$ & 0.41 & $1.07 \times 10^{9}$ \\
2 & PDMS & $1.03 \times 10^{3}$ & 0.48 & $1.2 \times 10^{9}$ \\
\hline
\end{tabular}

which is the complete conversion process of acoustic signals to electrical signals.

A group of multiple SNs respond to stimulus signals by forming sensory functional groups through specific innervation structures, which means the stimulus signals received by each sensory cell in a single SNM can be integrated and transmitted through nerve branches. Based on this bionic principle, annulus-shaped ciliary structure realizes the consistency of signal phases received by four units, which makes FUVH a linear additive array. Thus, the received signals can be directly added to facilitate algorithm processing (Fig. 4).

\section{Mechanical analysis}

\subsection{Sensing materials selection}

AFUVH is a resonant MEMS hydrophone, which mainly relies on the co-vibration of the sensing structure and the acoustic medium to receive acoustic signals. From vibrational science and hydroacoustics, to meet the conditions of picking up vibration [11,12], the average density of the annulus-shaped ciliary structure must be almost equal to that of water medium. Based on this principle, PE and PDMS firstly raise our attention, whose relevant properties are shown in Table 2 . The density of polyethylene (PE) and polydimethylsiloxane (PDMS) are both closed to that of water. Meanwhile, the appropriate toughness and rigidity of these two materials enable the external stress be transmitted easily. Therefore, PDMS is chosen to fabricate the rigid ring to form the cupula and PE is chosen to fabricate four cilia cylinders in this work.

\subsection{Properties of sensing structure}

Piezoresistive effect of monocrystal silicon is the sensitive mechanism of AFUVH, by which mechanical quantity is transferred into electrical signals. With the stress increase 


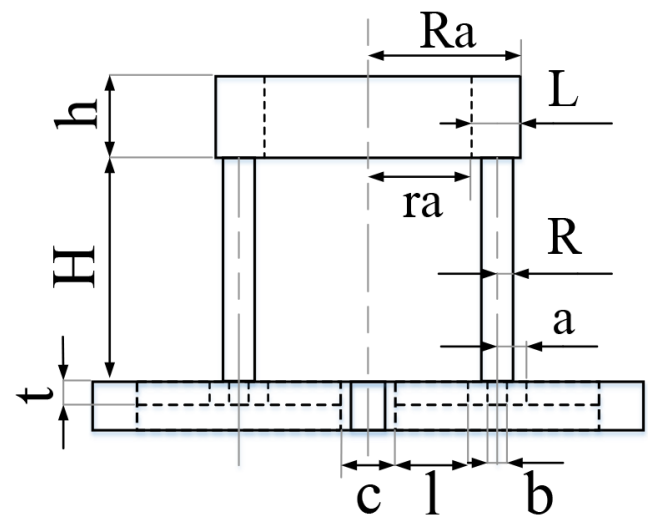

Fig. 5. Geometric parameter schematic of AFUVH.

on the cantilever beam, the resistance (rate) of piezoresistor changes more sharply along with the deformation of the piezoresistance. Then, the output voltage of Wheatstone bridge becomes much higher. That is to say the stress on the cantilever beam reflects the sensitivity of AFUVH.

According to previous research [13], the material mechanics principle and theoretical mechanics analysis, stress $\sigma_{(x)}$ at any point of a single cantilever beam is expressed as:

$$
\begin{aligned}
\sigma_{(x)}= & \frac{M}{W} \pm \frac{F_{H}}{b t}=\frac{l x}{(4+\varepsilon) l^{2}+12 a l+12 a^{2}+l(R a-0.5 L)} \\
& \times\left[\frac{\pi P R H^{2}}{4}+\frac{\pi P R a\left(H h+0.5 h^{2}\right)}{8}\right] \frac{1}{W} \\
& \pm \frac{\pi P R H+\pi P R a h}{b t}=\frac{3 \pi P L x}{4 b t^{2}} \\
& \times \frac{\left[2 R H^{2}+R a\left(H h+0.5 h^{2}\right)\right]}{(4+\varepsilon) l^{2}+(12 a+R a-0.5 L) l+12 a^{2}} \\
& \pm \frac{\pi P(R H+R a h)}{b t}
\end{aligned}
$$

Wherein $P$ is the external sound pressure and $\varepsilon=\frac{6 \beta}{1+\mu}$ ( $\beta$ is the coefficient of torsion of cantilever beam section, and $\mu$ is the Poisson's ratio of the cantilever beam). Different meanings of the parameters in formula (1) are shown in Figure 5. The parameters of the cross-beam in a single unit of FUVH are the same with previous work, which are shown in Table 3 [14]. Formula (1) indicates that the stress on the cross-beam rises with the radius and height of cilia cylinders, and the height of ring. That means the longer or thicker the cilia cylinders are and the higher the ring is, the higher sensitive of AFUVH is. It is also worth noting that, because of the ring, the barycentre of the whole sensing structure is projected on the center of the sensor chip, which is different from $\mathrm{CVH}$ whose barycentre of sensing structure is projected on the center mass block. Thus, there's an initial stress on the cantilever beam, which reduces the stress variation range of the cantilever beam in
Table 3. Parameters of cross beam.

\begin{tabular}{ll}
\hline Length of beams (l) & $1000 \mu \mathrm{m}$ \\
\hline Width of beams (b) & $120 \mu \mathrm{m}$ \\
Thickness of beams (t) & $40 \mu \mathrm{m}$ \\
Half length of the center block's side (a) & $300 \mu \mathrm{m}$ \\
\hline
\end{tabular}

the inner part of ring while increasing that in the outer part of ring.

Besides, the bandwidth is also an important feature of AFUVH performance, which is mainly decided by the natural frequency of the sensing structure. From previous study, with the increase of natural frequency, the bandwidth of AFUVH becomes wider. In addition, considering the application environment is water, the bandwidth of AFUVH is seriously affected by the fluidsolid coupling (FSI) of the sensing structure and water $[15,16]$. Thus, the static stress analysis and FSI analysis for AFUVH should be carried out further.

\section{Simulation optimization of AFUVH}

In this paper, by using ANSYS 17.0, the static analysis is carried out to indicate the maximum stress on $\mathrm{X}$ and $\mathrm{Y}$ direction cantilever beams, and FSI analysis is performed to indicate the natural frequency of AFUVH. Design variables are $\mathrm{H}$ (the height of cilia cylinders), D (the diameter of cilia cylinders), $\mathrm{h}$ (the height of ring) and $\mathrm{L}$ (the thickness of ring). Considering the restrictions of manufacturing process and sensor chip parameters, the design and optimization of annulus-shaped ciliary structure are performed by using the following parameter ranges: (1) $2 \mathrm{~mm} \leq \mathrm{H} \leq 5 \mathrm{~mm}$; (2) $0.2 \mathrm{~mm} \leq \mathrm{D} \leq 0.5 \mathrm{~mm}$; (3) $0.5 \mathrm{~mm} \leq \mathrm{h} \leq 3 \mathrm{~mm}$; (4) $0.2 \mathrm{~mm} \leq \mathrm{L} \leq 1 \mathrm{~mm}$; (5) FSI frequency $\mathrm{f}>1000 \mathrm{~Hz}$. Figures 6 and 7 show the simulation results, and Figure 8 shows the impact of the variable parameters.

As shown in Figure 6, the stress on the cantilever beam rises with the increase of $\mathrm{H}$ and h. In Figure 7, except for diameter D, all other parameters have the negative relationship with the sensing structure natural frequency. Figure 8 shows the influence of different parameterson natural frequency and maximum stress, which indicates that the stress on the cantilever beam and the sensing structure natural frequency are a pair of contradictory features. That is to say the sensitivity and the bandwidth of AFUVH are also a pair of contradictory features.

From Figure 8, the parameter D has the great positive influence on natural frequency and the little negative influence on maximum stress on the cantilever beams, while the parameter L are totally on the contrary. Therefore, changing two specific parameters D and L is sufficient to solve the problem above. Then, selecting the appropriate combination of different parameter sizes is of great importance. Two design purposes of the simulation optimization progress are shown as follows: (1) AFUVH has the better sensitivity than FUVH; (2) AFUVH retains its bandwidth more than $1000 \mathrm{~Hz}$ as much as possible. 

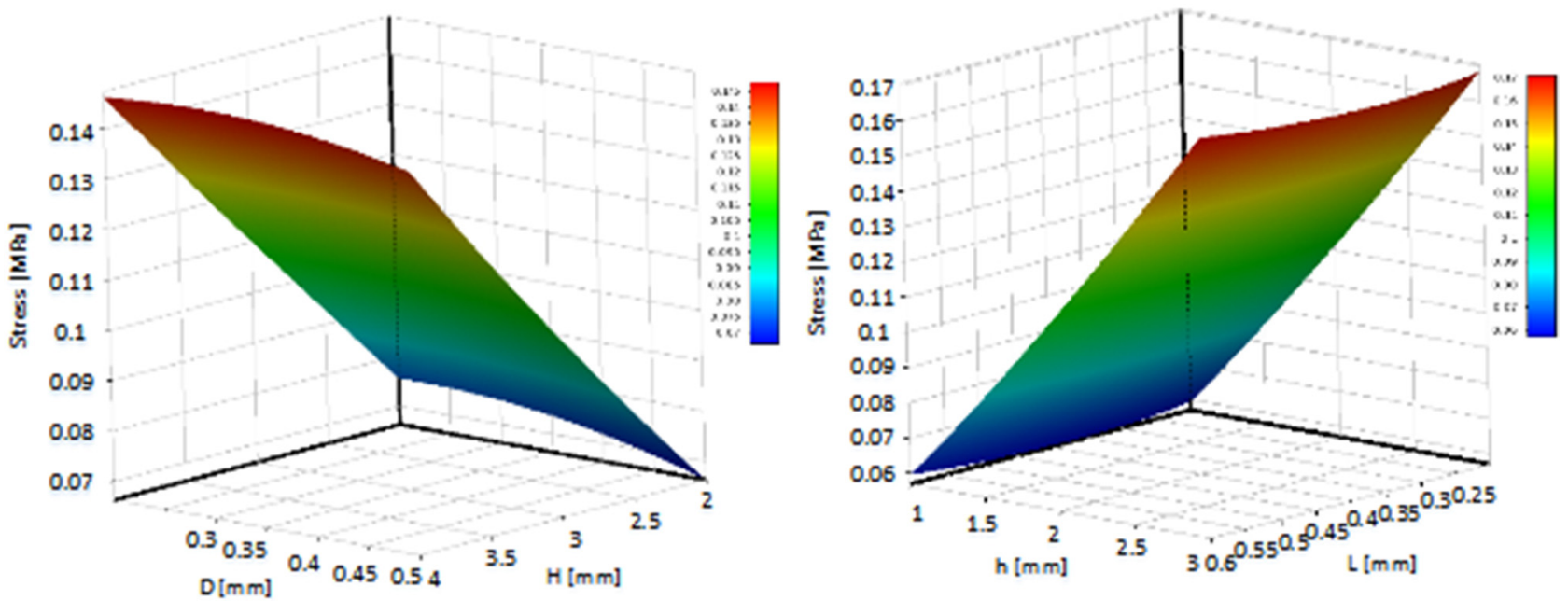

Fig. 6. The relationship between parameters $(H, h, R, L)$ and maximum stress on the cantilever beam.
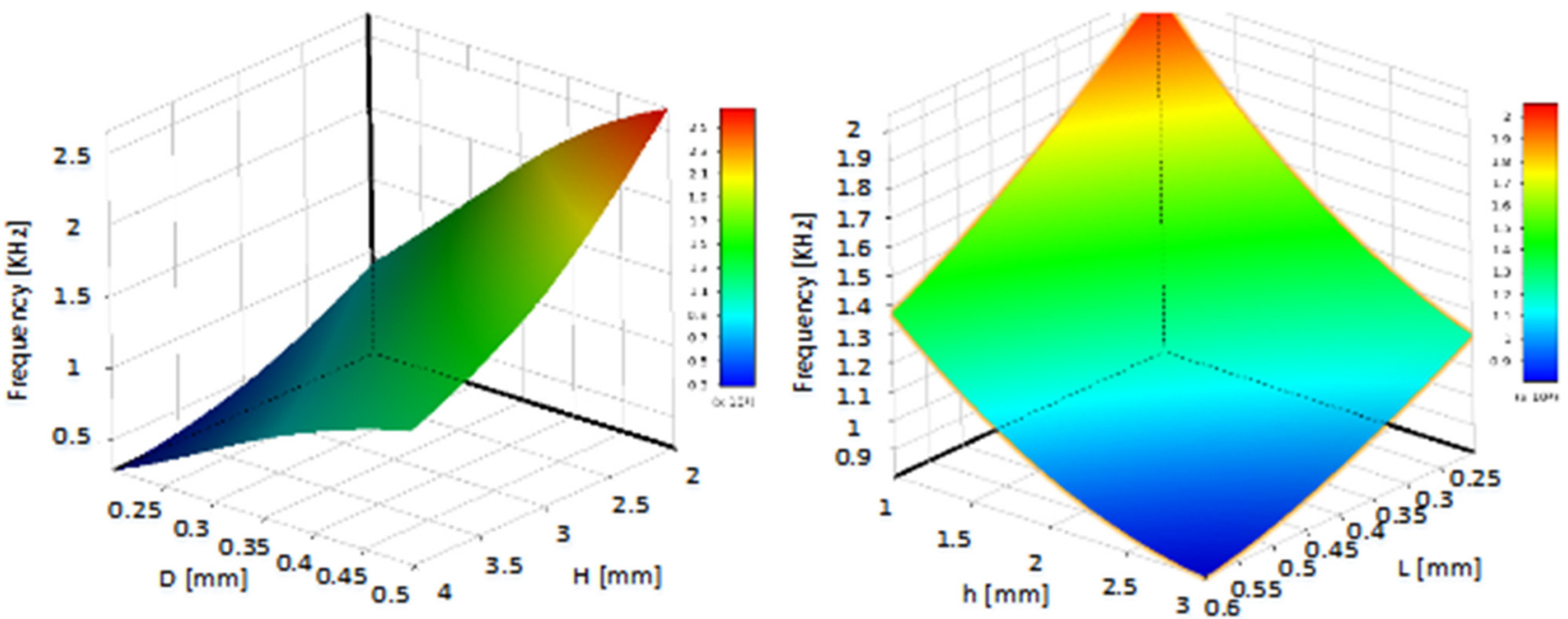

Fig. 7. The relationship between parameters $(H, h, R, L)$ and natural frequency.

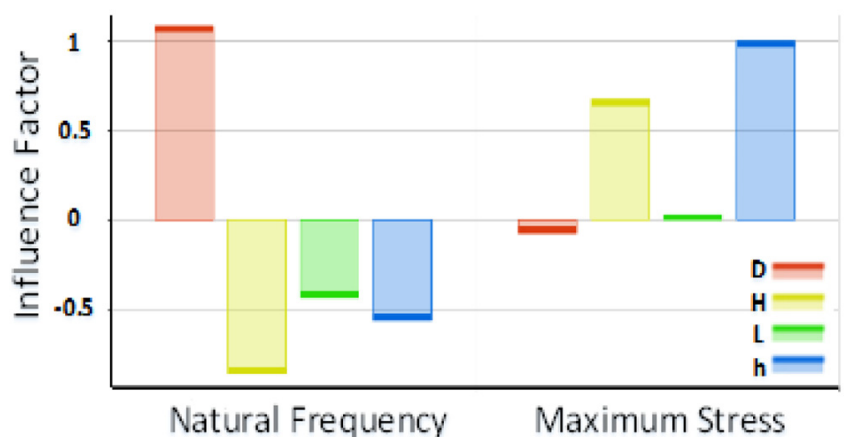

Fig. 8. The impact of parameters $(H, h, R, L)$ on natural frequency and maximum stress on the cantilever beams.

Before determining the specific parameter sizes, establishing a preliminary model of AFUVH and carrying out a static analysis of this model, the stress nephogram is shown in Figure 9a and the stress curves of this preliminary model are shown in Figure 9b. Especially in Figure 9b, it can be seen from the stress curves of four beams in the $\mathrm{X}$ direction that the maximum stresses on cantilever beams (1) and (4) are obviously greater than those on cantilever beams (2) and (3). That is to say the stress variation range of the cantilever beams in the inner part of ring is wider than that in the outer part of ring, which is consistent with the mechanical analysis in PART 3.2. Because of the great consistency of sensor chip produced by MEMS process, the resistances of piezoresistors on each cantilever beam are almost same, which means any two cantilever beams in the same direction can be combined to form a Wheatstone bridge. Thus, this problem can be solved by adjusting the combination of cantilever beams. Figure 10 shows the overall FUVH sensor chip. Finally, AFUVH can be combined to four rearranged sensor units (two sensor units with high sensitivity as shown in Fig. 10a, and two with low sensitivity as shown in Fig. 10b), which is different from cilium-shaped FUVH. 


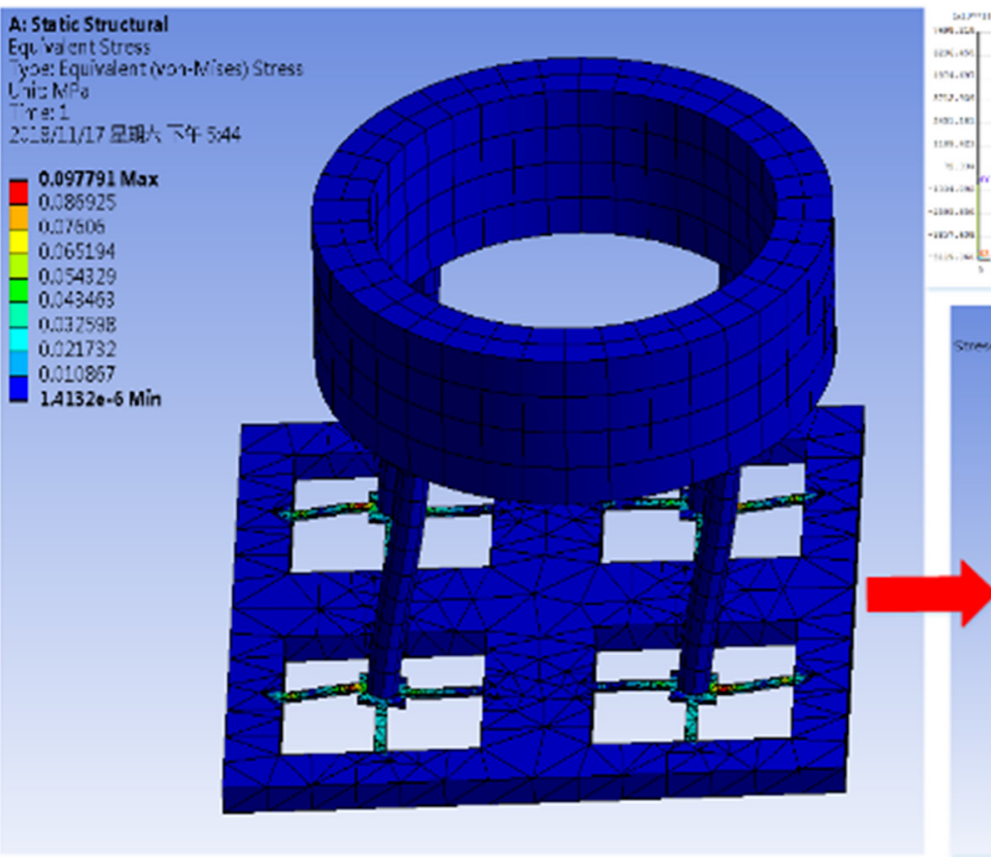

(a)
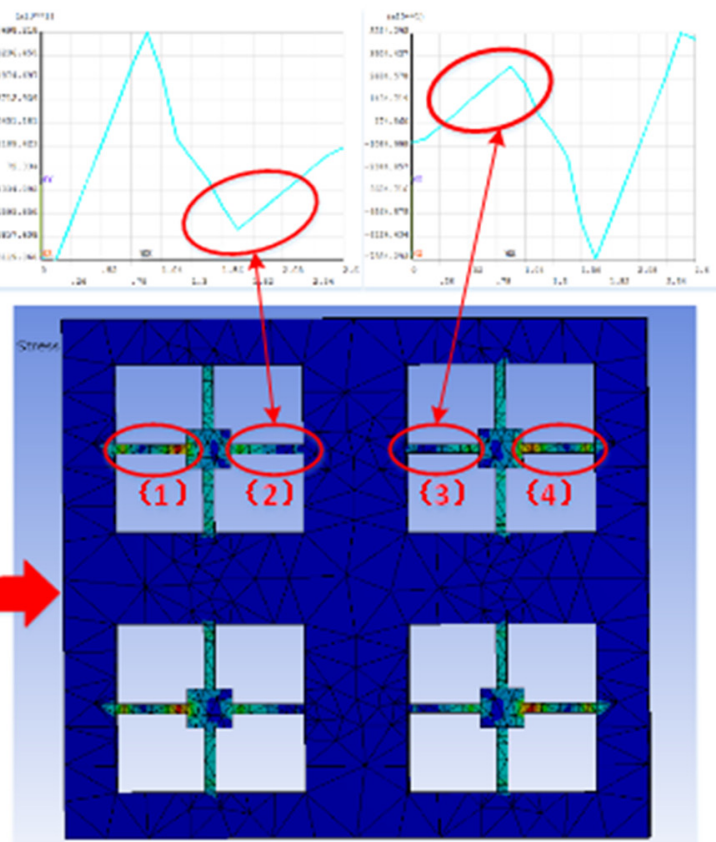

(b)

Fig. 9. Stress nephogram and stress curves of AFUVH. (a) Stress nephogram (b) Stress curves of the cantilever beams.
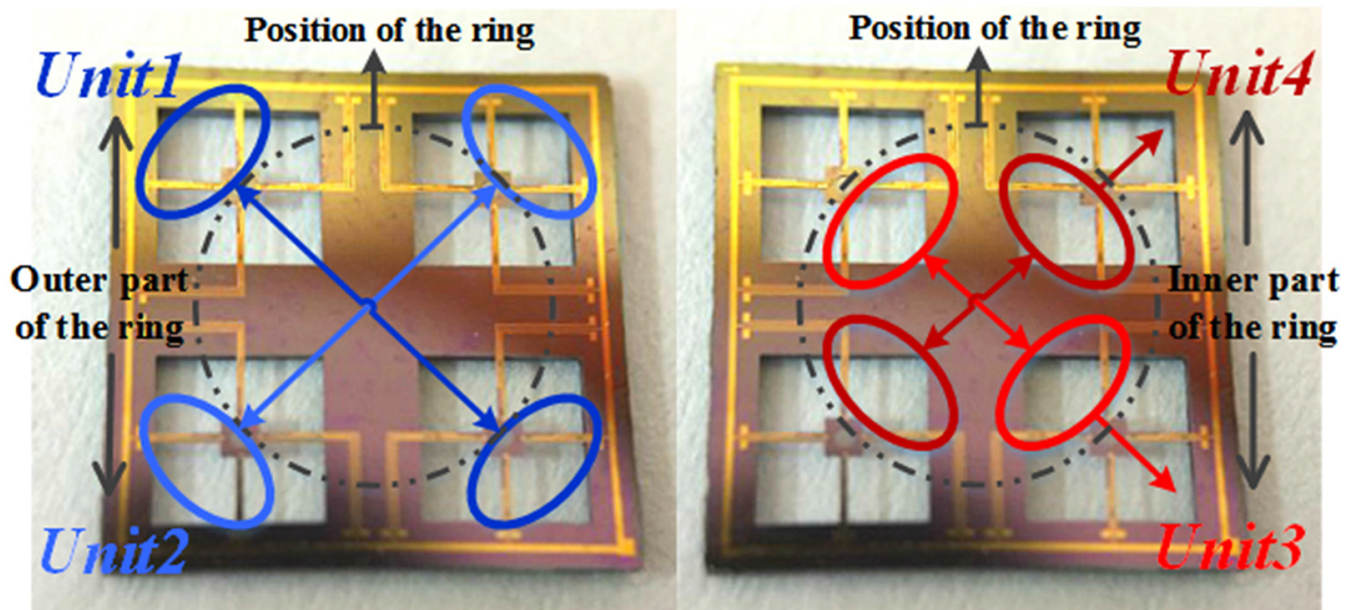

Fig. 10. New combination of AFUVH sensor units.

Comparing this new sensing structure with ciliumshaped FUVH, the cilium radius of both structures should be invariant. Meanwhile, to ensure the improvement of AFUVH performance, the maximum stress on the cantilever beam in the inner part of ring should be not less than that of FUVH. On these premises, the parameter sizes of AFUVH basic model are determined in this paper: $\mathrm{H}=3 \mathrm{~mm}, \mathrm{D}=0.4 \mathrm{~mm}, \mathrm{~h}=1.75 \mathrm{~mm}$ and $\mathrm{L}=0.72 \mathrm{~mm}$. In this model, the maximum stress on the cantilever beam in the inner part of the ring is closely equal to that of FUVH. Its design purpose is to demonstrate that AFUVH has better performance than FUVH in receiving acoustic signals.
However, this basic model mainly takes the former design purpose into consideration, which is not comprehensive. Therefore, based on this model, an optimized model of AFUVH is designed by using the multi-parameter optimization function of ANSYS 17.0 workbench and taking the influence factors and influence trends of each parameter as references. The parameter sizes of AFUVH optimized model are finally determined: $\mathrm{H}=3 \mathrm{~mm}$, $\mathrm{D}=0.4 \mathrm{~mm}, \mathrm{~h}=2 \mathrm{~mm}$ and $\mathrm{L}=0.2 \mathrm{~mm}$. This optimized model is used to demonstrate what the best effect AFUVH can achieve.

To ensure the reliability of the optimized design method, by using these two model parameters, the static 
stress analysis and FSI analysis are performed in the following text.

Figure 11 shows the stress contrast curves on the cantilever beam of FUVH, AFUVH basic model, and

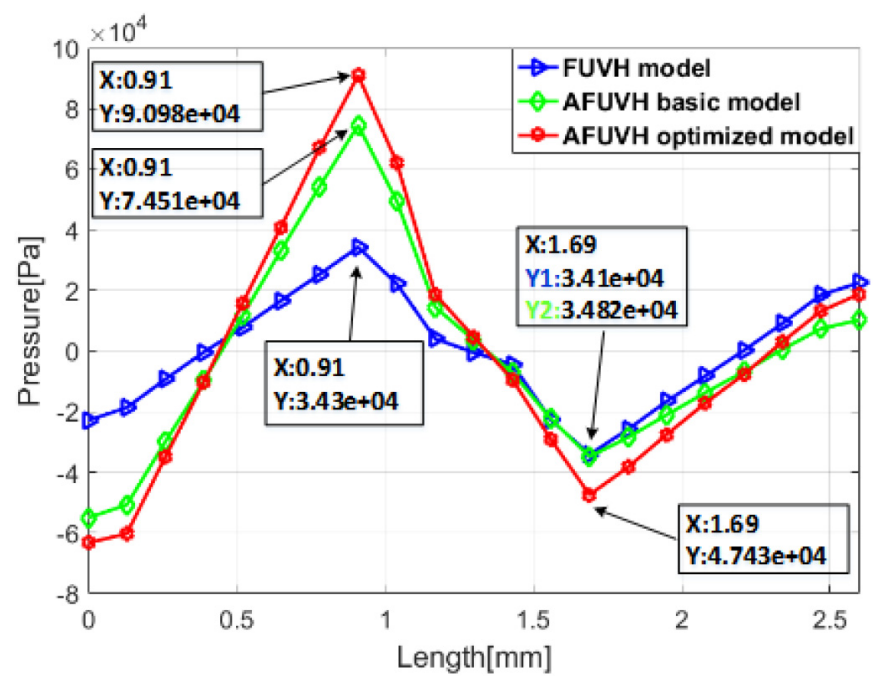

Fig. 11. The stress contrast diagram on the cantilever beams.
AFUVH optimized model. Comparing with FUVH, the maximum stress on the cantilever beam of the AFUVH basic model rises up to 2.17 times, which means the sensitivity of a high-sensitivity unit is increased by $6.73 \mathrm{~dB}$. Meanwhile, the maximum stress on the cantilever beam of the AFUVH optimized model rises up to 2.65 times, which means the sensitivity of a high-sensitivity unit of AFUVH can be improved by $8.16 \mathrm{~dB}$. Especially in this structure, the maximum stress on the cantilever beam in the inner part of the ring is 1.39 times that of previous one, which means the sensitivity of a low-sensitivity unit of AFUVH can also be improved by $2.86 \mathrm{~dB}$. In conclusion, the annulus-shaped ciliary structure for FUVH is able to improve the sensitivity of previous cilium-shaped sensing structure for FUVH effectively.

Figure 12 shows the results of FSI analysis. The wet mode response results of AFUVH are both more than $1000 \mathrm{~Hz}$ (The first-order wet mode response of AFUVH basic model and AFUVH optimized model reaches up to 1003.93 and $1216.62 \mathrm{~Hz}$, respectively), which achieves the original design purposes. Therefore, the annulus-shaped ciliary structure can improve the performance of FUVH. Then, a prototype of AFUVH are fabricated and tested experimentally.

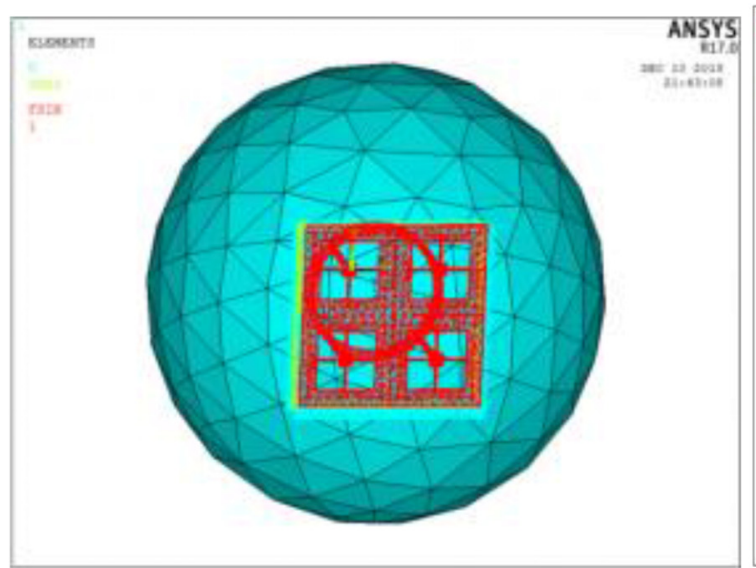

(a)

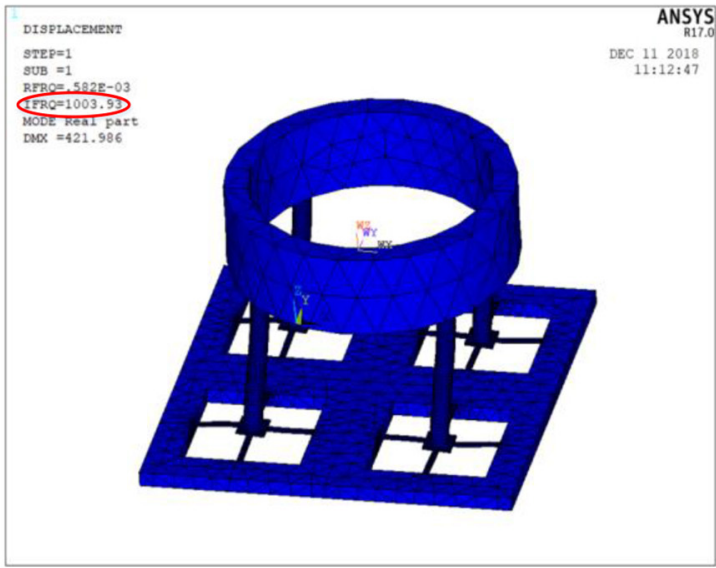

(b)

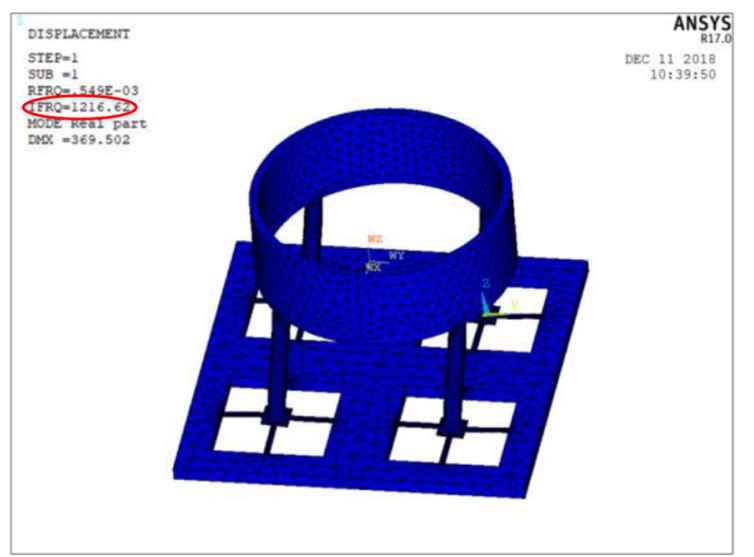

(c)

Fig. 12. The analysis of FSI. (a) Fluid-solid coupling interface (b) AFUVH basic model (c) AFUVH optimized model. 


\section{Experiments for AFUVH}

\subsection{Fabrication}

\subsubsection{Fabrication of sensor chips}

Four-unit sensor chips are fabricated by MEMS silicon micro-machining technology, which mainly use the bulk micro-machining technology and silicon bond binding technology. The process includes lithography, oxidation, bonding, etching, ion implantation, film growth, evaporation and so on. Four-unit sensor chips are finally formed after deep silicon etching, wet etching and other processes. Figure 13 shows the SEM image of FUVH sensor chip.

\subsubsection{Consistency test of four-unit sensor chip}

According to Section 4, Wheatstone bridges formed by the piezoresistors on cantilever beams need to be recombined, which requires great consistency of the piezoresistors manufactured by MEMS processing technology. Thus, it is necessary to test each value of 32 piezoresistors distributed on sixteen cantilever beams.

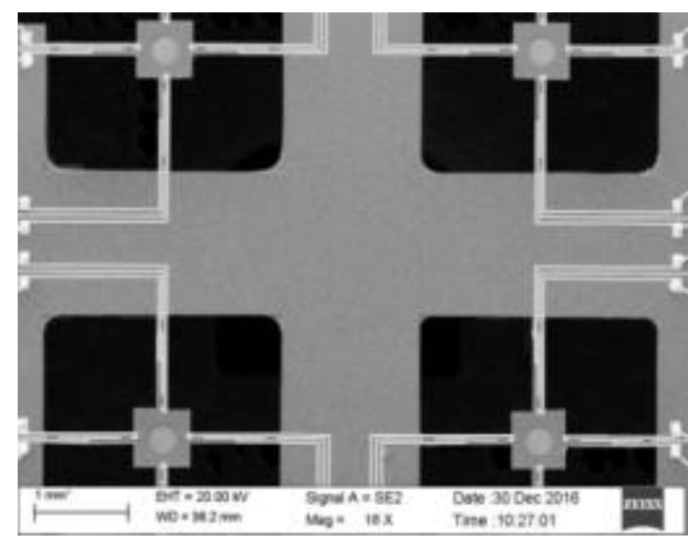

Fig. 13. SEM image of FUVH sensor chip.

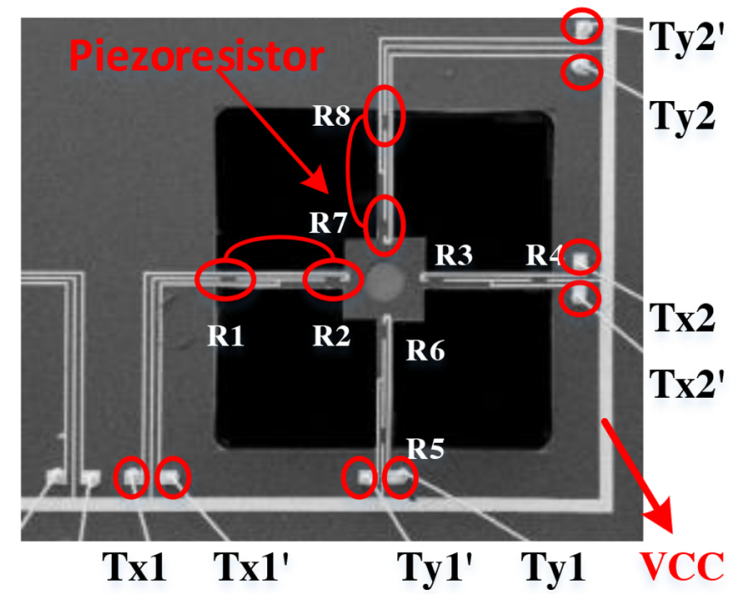

(a)
Taking a unit of FUVH chip as an example, the distribution of piezoresistors on cantilever beams is shown in Figure 14a. Tx1, Tx1, Tx2, Tx2 and Ty1, Ty1, Ty2, Ty2 respectively represent the resistance testing points ("X" means X-direction and "Y" means Y-direction). Figure 14b shows the connection diagram of equivalent resistances, in which the design value of each equivalent resistance is $\mathrm{R}=500 \Omega$. Thus, the equivalent resistance between VCC and $T x 1$ can be represented as $R_{V c c-T x 1}$, and the rest equivalent resistances between $\mathrm{VCC}$ and other testing points are represented in the same way. Theoretically, the design value of $R_{V c c-T x 1}, R_{V c c-T x 2}, R_{V c c-T y 1}, R_{V c c-T y 2}$ should be $2 \mathrm{R}=1000 \Omega$ and $R_{V c c-T x 1}, R_{V c c-T x 2}, R_{V c c-T y 1}$, $R_{V c c-T y 2}$ should be $\mathrm{R}=500 \Omega$. The testing value of equivalent resistances are shown in Table 4 .

From Table 4 , the testing value of $2 \mathrm{R}$ is about $1000 \pm 31$ $\Omega$ and $\mathrm{R}$ is about $500 \pm 16 \Omega$, which means the maximum inconsistency (maximum error of $\mathrm{R}$ ) of piezoresistors on cantilever beams is about $3.2 \%$. In conclusion, the FUVH sensor chip is of good consistency.

\subsubsection{Fabrication of sensing structure}

Considering the accuracy of the annulus-shaped ciliary structure fabricating process, an accurate casting model method is adopted. Firstly, infusing the PDMS into a mold, after the solidification of the PDMS, the rigid ring is completed. Secondly, another key fabrication procedure is the integration of the rigid ring, four cilia cylinders and four center blocks of sensor chip. Through the dedicated integration platform, the UV-curable adhesive is adopted to attach four cilia cylinders to the rigid ring. Then, the integration of the anulus-shaped ciliary structure and cross beam is performed in the same way. Thirdly, by taking the way of vacuum vapor deposition, the sensing structure and the four-unit chip are coated with a parylene membrace layer to ensure its overall insulativity $[17,18]$. Figures $15 \mathrm{a}-15 \mathrm{e}$ shows the fabrication process of the sensing structure and the integrating progress. Figures 16a and $16 \mathrm{~b}$ shows the actual progresses. 
Table 4. Testing value of equivalent resistances.

\begin{tabular}{llcccc}
\hline No. $\Omega$ & & Unit-I & Unit-II & Unit-III & Unit-IV \\
\hline \multirow{4}{*}{ X direction } & $R_{V c c-T x 1^{\prime}}$ & 514 & 502 & 508 & 510 \\
& $R_{V c c-T x 2^{\prime}}$ & 515 & 512 & 509 & 512 \\
& $R_{V c c-T x 1}$ & 1020 & 1031 & 1020 & 1024 \\
& $R_{V c c-T x 2}$ & 1025 & 1030 & 1017 & 1015 \\
Y direction & $R_{V c c-T y 1^{\prime}}$ & 507 & 503 & 516 & 506 \\
& $R_{V c c-T y 2^{\prime}}$ & 505 & 516 & 508 & 515 \\
& $R_{V c c-T y 1}$ & 1017 & 1028 & 1013 & 1026 \\
& $R_{V c c-T y 2}$ & 1024 & 1016 & 1027 & 1016 \\
\hline
\end{tabular}

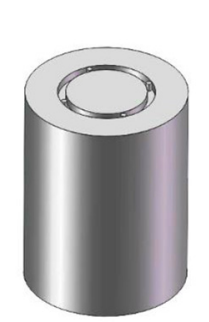

(a)

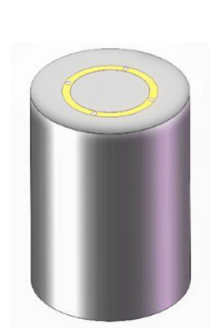

(b)

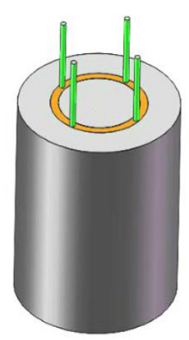

(c)

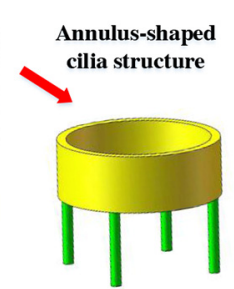

(d)

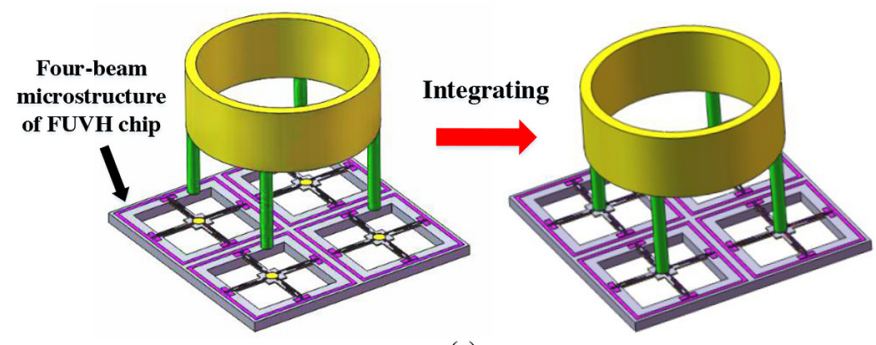

(e)

Fig. 15. Integration process of the sensing structure.

AFUVH is applied to different depth of underwater environment, so it should be encapsulated in a stainlesssteel shell from being damaged by the hydraulic pressure. Low-noise preamplifier circuit and the adding circuit are also placed in this shell to pre-process electrical signals from different Wheatstone bridges. Figure 17 shows the material object of AFUVH.

\subsection{Experiments}

A specific calibration system is used to test the sensitivity of AFUVH. Figure 18a shows the schematic diagram of calibration system. Figure $18 \mathrm{~b}$ shows the experimental testing environment. The method of comparative calibration in the standing wave tube is still adopted for the test of sensitivity [19]. The sensitivity can be expressed as:

$$
M_{x}=20 \lg \left(\frac{V_{x}}{V_{0}} \frac{\sin k d}{\cos k d_{0}}\right)+M_{0}
$$

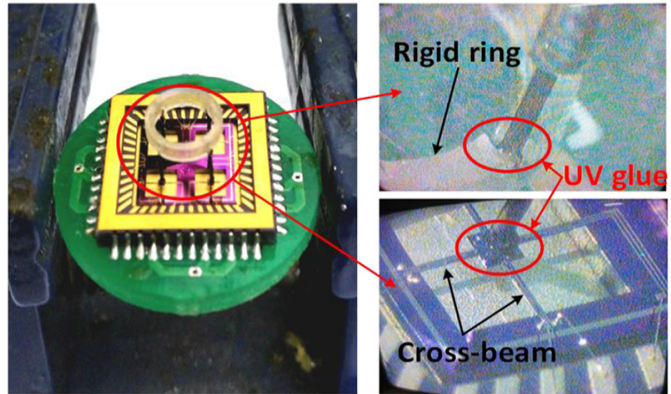

(a) Integration of the ring and the cilia

(b) Integration of the crossbeam and the cilia

Fig. 16. Integration progress diagram.

In formula (2), $M X$ represents the sensitivity of the tested AFUVH; $M_{0}$ represents the sensitivity of a standard hydrophone (standard hydrophone's sensitivity is $-170 \mathrm{~dB}) ; V_{O}$ and $V_{X}$ respectively represent the output voltage of AFUVH and that of the standard hydrophone; $k$ is the wave number; $d$ and $d_{0}$ respectively represent the distance from tested AFUVH and standard hydrophone to water surface. According to the sensitivity gain analysis of multiple sensor units vector hydrophone [5], through calculation, frequency response comparison diagram of the three structures are shown in Figure 19.

\section{Discussion}

Comparing with cilium-shaped FUVH, the sensitivity of AFUVH basic model can be improved by $3.92 \mathrm{~dB}$ at $1000 \mathrm{~Hz}$, in addition, the sensitivity of AFUVH optimized model can be improved by $5.87 \mathrm{~dB}$ at $1000 \mathrm{~Hz}$. And there are similar improvements at $20-1000 \mathrm{~Hz}$. These experimental results are consistent with the theory and simulation. Moreover, the working bandwidth of these two model are both above $1000 \mathrm{~Hz}$, which is consistent with the results of FSI simulation and meets the original design requirement. It has been proved that AFUVH has better characteristics than FUVH, but its fabrication process is more complex. The ring structure must be precise and the anulus-shaped ciliary structure must be integrated accurately. 


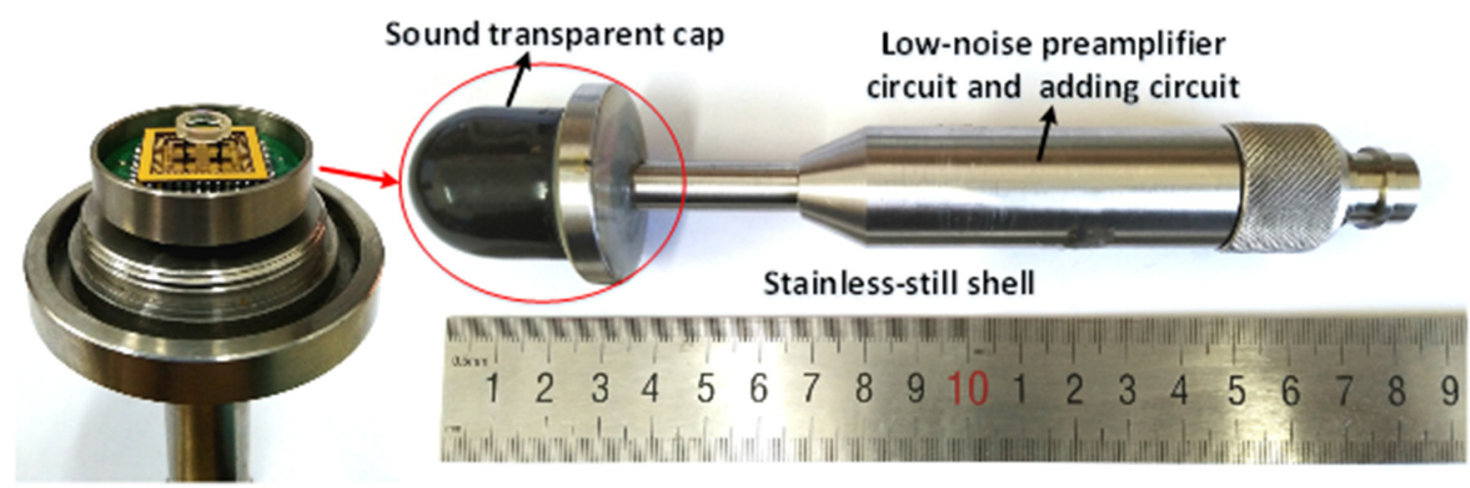

Fig. 17. Material object of hydrophone.

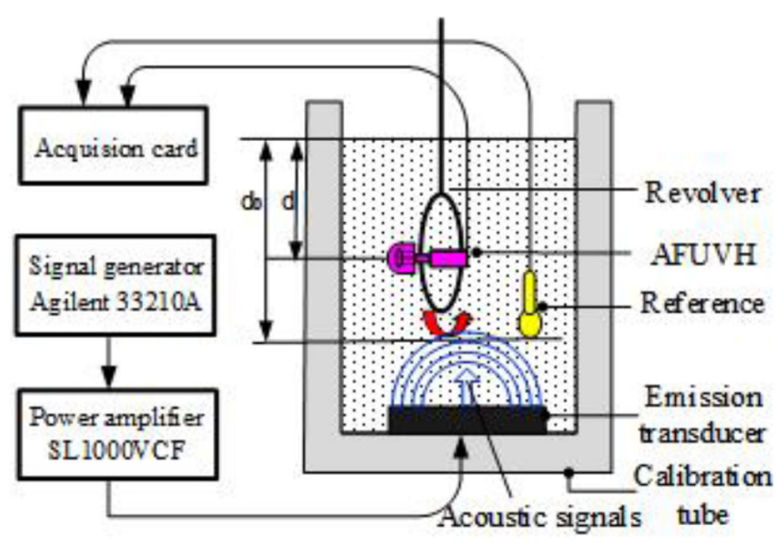

(a)

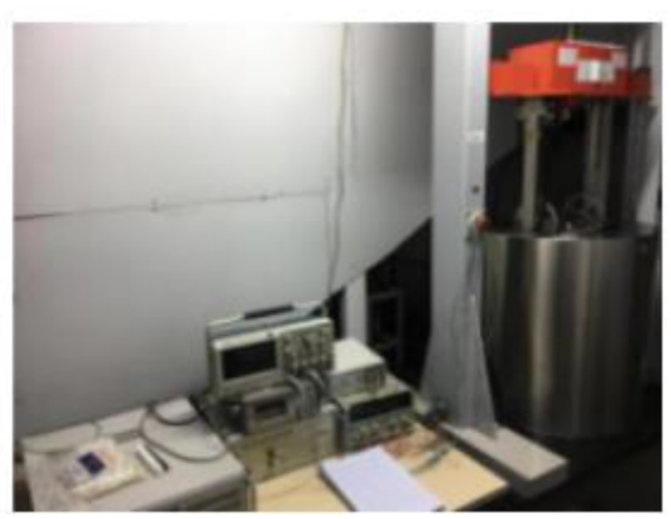

(b)

Fig. 18. Experimental test picture. (a) Schematic diagram of calibration system (b) Experimental testing environment.

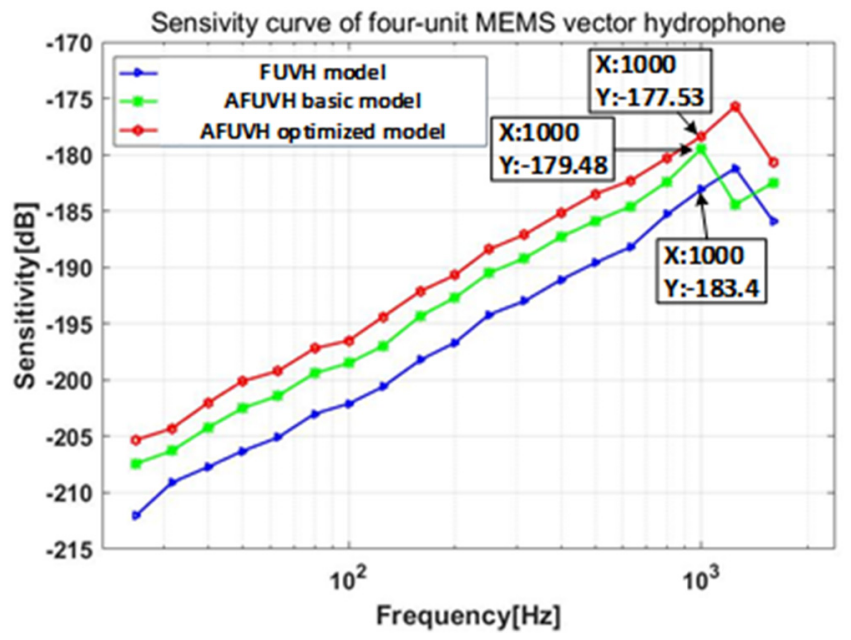

Fig. 19. Sensitivity curve of hydrophone.

\section{Conclusion}

In this paper, anulus-shaped ciliary structure for four-unit MEMS vector hydrophone changes the application situation where each unit of four-unit sensor chip is mutually independent. Inspired by bionics, this innovative structure realizes the complete simulation of the lateral line neuromasts of the blind cave fish. By mimicking the biological structure "cupula", this structure enhances the sensitivity and ensures the appropriate bandwidth at the same time, which improves the performance of four-unit MEMS vector hydrophone. The above judgment is supported by theory, simulation and experiment. The sensitivity and working frequency of AFUVH is measured accurately in the lab. The sensitivity of AFUVH can reach up to $3.92 \mathrm{~dB}$ with effective structure and $5.87 \mathrm{~dB}$ with optimum dimension structure at $1000 \mathrm{~Hz}$, and satisfies the increments of $6 \mathrm{~dB}$ per octave. Meanwhile, the range of AFUVH working frequency is $20-1000 \mathrm{~Hz}$. In conclusion, anulus-shaped ciliary structure is a satisfactory design for four-unit MEMS vector hydrophone, also providing a great reference for further research and application of multi-unit MEMS vector hydrophone.

This work was supported by National Natural Science Foundation of China (61525107, 51875535, 61927807), National Key Research and Development Project (2019YFC0119800), The Fund for Shanxi '1331 Project' Key Subject Construction and Innovation Special Zone Project, The Science and Technology Innovation Project of Shanxi Higher Education Institution 
(2020L0718), The Teaching Reform Project in Higher School in Shanxi (J2020383), Cooperative education project of industry university cooperation (201901157012).

\section{References}

1. C.Y. Xue, S. Chen, W.D. Zhang, B.Z. Zhang, G.J. Zhang, H. Qiao, Design, fabrication, and preliminary characterization of a novel MEMS bionic vector hydrophone, Microelectron. J. 38, 1021-1026 (2007)

2. S. Hanasoge, M. Ballard, P.J. Hesketh, A. Alexeev, Asymmetric motion of magnetically actuated artificial cilia, Lab on A Chip 17, 3138-3145 (2018)

3. A.G.P. Kottapalli, M. Bora, E. Kanhere, M. Asadnia, J.M. Miao, M.S. Triantafyllou, Cupula-inspired hyaluronic acidbased hydrogel encapsulation to form biomimetic MEMS flow sensors, Sensors 17, 14 (2017)

4. G.J.M. Krijnen, M. Dijkstra, J.J. van Baar, S.S. Shankar, W. J. Kuipers, R.J.H. de Boer, D. Altpeter, T.S.J. Lammerink, R. Wiegerink, MEMS based hair flow-sensors as model systems for acoustic perception studies, Nanotechnology 17, S84-S89 (2006)

5. X.Y. Zhang, Q.D. Xu, G.J. Zhang, N.X. Shen, Z.Z. Shang, Y. Pei, J.W. Ding, L.S. Zhang, R.X. Wang, W.D. Zhang, Design and analysis of a multiple sensor units vector hydrophone, AIP Adv. 8, 9 (2018)

6. Z. Xiaoyong, L. Laiyuan, Ship radiated noise detector when line spectrum parameters are unknown, Acta Acust. 40, 511-518 (2015)

7. W. Xu, Y. Liu, G.J. Zhang, R.X. Wang, C.Y. Xue, W.D. Zhang, J. Liu, Development of cup-shaped microelectromechanical systems-based vector hydrophone, J. Appl. Phys. 120, 8 (2016)

8. M. Bora, A.G.P. Kottapalli, J.M. Miao, M.S. Triantafyllou, Fish-inspired self-powered microelectromechanical flow sensor with biomimetic hydrogel cupula, APL Mater. 5, 6 (2017)
9. Y. Liu, R.X. Wang, G.J. Zhang, J. Du, L. Zhao, C.Y. Xue, W.D. Zhang, J. Liu, "Lollipop-shaped" high-sensitivity Microelectromechanical Systems vector hydrophone based on Parylene encapsulation, J. Appl. Phys. 118, 7 (2015)

10. A. Nagiel, D. Andor-Ardo, A.J. Hudspeth, Specificity of afferent synapses onto plane-polarized hair cells in the posterior lateral line of the zebrafish, J. Neurosci. 28, 8442-8453 (2008)

11. M.B. Moffett, D.H. Trivett, P.J. Klippel, P.D. Baird, A piezoelectric, flexural-disk, neutrally buoyant, underwater accelerometer, IEEE Trans. Ultrason. Ferroelectr. Fr eq. Control 45, 1341-1346 (1998)

12. V.A. Shchurov, V.I. Ilyichev, V.P. Kuleshov, M.V. Kuyanova, The interaction of energy flows of underwater ambient noise and a local source, J. Acoust. Soc. Am. 90, 1002-1004 (1991)

13. B.Z. Zhang, H. Qiao, S. Chen, J. Liu, W.D. Zhang, J.J. Xiong, C.Y. Xue, G.J. Zhang, Modeling and characterization of a micromachined artificial hair cell vector hydrophone, Microsyst. Technol. 14, 821-828 (2008)

14. M.R. Liu, G.J. Zhang, X.P. Song, Y. Liu, W.D. Zhang, Design of the monolithic integrated array MEMS hydrophone, IEEE Sens. J. 16, 989-995 (2016)

15. G.J. Zhang, P. Zhao, W.D. Zhang, Resonant frequency of the silicon micro-structure of MEMS vector hydrophone in fluidstructure interaction, AIP Adv. 5, 8 (2015)

16. C. Vančura, I. Dufour, S.M. Heinrich, F. Josse, A. Hierlemann, Analysis of resonating microcantilevers operating in a viscous liquid environment, Sens. Actuators A Phys. 141, 43-51 (2008)

17. A. Qualtieri, F. Rizzi, G. Epifani, A. Ernits, M. Kruusmaa, M.D. Vittorio, Parylene-coated bioinspired artificial hair cell for liquid flow sensing, Microelectron. Eng. 98, 516-519 (2012)

18. N. Chen, C. Tucker, J.M. Engel, Y. Yang, C. Liu, Design and characterization of artificial haircell sensor for flow sensing with ultrahigh velocity and angular sensitivity, J. Microelectromech. Syst. 16, 999-1014 (2007)

19. W.D. Zhang, L.G. Guan, G.J. Zhang, C.Y. Xue, K.R. Zhang, J.P. Wang, Research of DOA estimation based on single MEMS vector hydrophone, Sensors 9, 6823-6834 (2009)

Cite this article as: Xiaoyong Zhang, Nixin Shen, Qingda Xu, Yu Pei, Yuqi Lian, Weidong Wang, Guojun Zhang, Wendong Zhang, Design and implementation of anulus-shaped ciliary structure for four-unit MEMS vector hydrophone, Int. J. Metrol. Qual. Eng. 12, $4(2021)$ 